\title{
Cellular fate of intersex differentiation
}

\author{
Xin Wang ${ }^{1}$, Fengling Lai', Dantong Shang ${ }^{1}$, Yibin Cheng ${ }^{1}$, Tian Lan ${ }^{1}$, Hanhua Cheng ${ }^{1}$ and Rongjia Zhou (1)
}

\begin{abstract}
Infertile ovotestis (mixture of ovary and testis) often occurs in intersex individuals under certain pathological and physiological conditions. However, how ovotestis is formed remains largely unknown. Here, we report the first comprehensive single-cell developmental atlas of the model ovotestis. We provide an overview of cell identities and a roadmap of germline, niche, and stem cell development in ovotestis by cell lineage reconstruction and a uniform manifold approximation and projection. We identify common progenitors of germline stem cells with two states, which reveal their bipotential nature to differentiate into both spermatogonial stem cells and female germline stem cells. Moreover, we found that ovotestis infertility was caused by degradation of female germline cells via liquid-liquid phase separation of the proteasomes in the nucleus, and impaired histone-to-protamine replacement in spermatid differentiation. Notably, signaling pathways in gonadal niche cells and their interaction with germlines synergistically determined distinct cell fate of both male and female germlines. Overall, we reveal a cellular fate map of germline and niche cell development that shapes cell differentiation direction of ovotestis, and provide novel insights into ovotestis development.
\end{abstract}

\section{Introduction}

Why and how sex is formed and maintained in two sexes, male and female, in most living organisms, have remained key questions in biology for over a century. Sexual reproduction exists in nearly all animals. In general, sex, as such a fundamental aspect of life, is nearly divided into male and female. However, the phenotype can be blurred in humans and other animals. Gynandromorph and intersex can happen in the same individual owing to, for example, genetic aberrations and/or developmental defects, in which the sex identity of cells is mixed together. In humans, patients with disorders of sex development (DSD) are often considered an ambiguity of phenotypic sex. DSDs are congenital conditions in which the development of chromosomal, gonadal, or anatomical sex is atypical ${ }^{1}$. Worldwide, genital ambiguity occurs in one in 2000-4500 in humans ${ }^{2}$; however, only approximately one-fifth of the DSDs can be diagnosed by

\footnotetext{
Correspondence: Hanhua Cheng (hhcheng@whu.edu.cn) or Rongjia Zhou (rjzhou@whu.edu.cn)

${ }^{1}$ Hubei Key Laboratory of Cell Homeostasis, College of Life Sciences, Renmin Hospital of Wuhan University, Wuhan, China

Edited by A. Stephanou
}

molecular approaches ${ }^{3}$. Patients with DSDs are not only infertile, but also suffer from many health problems, including other malformations, developmental delay, intellectual impairment, and mental and psychosocial problems ${ }^{1}$. Understanding of sexual determination and differentiation mechanisms will benefit both diagnostic testing and medical therapy of DSDs.

In mammals, $S R Y$ on the Y chromosome is a dominant gene for male development ${ }^{4}$. In the embryonic gonads of males, SRY, together with NR5A1, upregulates SOX9 expression via its enhancer ${ }^{5,6}$; thus, a developmental pathway for testis formation is initiated. Otherwise, in the absence of $S R Y$, ovary development will start via several female pathways. RSPO1/WNT signaling is a major pathway for ovary development, which includes RSPO1, WNT4B, CTNNB1 and GSK3A/B $\mathrm{B}^{7,8}$. Mutations in these sex-determining genes and the dysregulation of relevant pathways will lead to DSDs, including 46,XY complete or partial gonadal dysgenesis, 46,XX testicular DSD, and $46, \mathrm{XX}$ ovotesticular DSD. For example, mutations in the conserved HMG-box and both $5^{\prime}$ and $3^{\prime}$ regions of sexdetermining gene $S R Y$ caused $\mathrm{XY}$ gonadal dysgenesis/sex reversal, in which the ovarian structure was often in a

\section{(c) The Author(s) 2021}

(c) (i) Open Access This article is licensed under a Creative Commons Attribution 4.0 International License, which permits use, sharing, adaptation, distribution and reproduction cc) in any medium or format, as long as you give appropriate credit to the original author(s) and the source, provide a link to the Creative Commons license, and indicate if changes were made. The images or other third party material in this article are included in the article's Creative Commons license, unless indicated otherwise in a credit line to the material. If material is not included in the article's Creative Commons license and your intended use is not permitted by statutory regulation or exceeds the permitted use, you will need to obtain permission directly from the copyright holder. To view a copy of this license, visit http://creativecommons.org/licenses/by/4.0/. 
degenerate state ${ }^{9-12}$. Mutations of WT1, NR5A1, and FOG2 caused 46,XX testicular/ovotesticular DSDs ${ }^{13-15}$. A missense mutation in the MAP3K1 gene, which reduced SOX9 expression of the male pathway and increased CTNNB1 activity in the female pathway, led to $46, \mathrm{XY}$ complete gonadal dysgenesis ${ }^{16}$. Deletions/point mutations of NR2F2 led to 46,XX ovotesticular DSD ${ }^{17,18}$. Gene duplications in sex-determining pathways can also lead to ovotesticular DSD. For example, a duplication of $1114 \mathrm{~kb}$ in the region of $17 \mathrm{q} 24.3$ containing SOX9 was associated with SRY negative 46,XX ovotesticular $\mathrm{DSD}^{19}$, and duplications of SOX3 also affected gonad development and resulted in 46,XX ovotesticular DSD in $S R Y$-negative patients $^{20,21}$. In addition, gonadal dysgenesis had a high risk for germ cell cancer formation ${ }^{22}$. Notably, mosaic karyotypes with three cell types, 46,X,dic $(\mathrm{X} ; \mathrm{Y})(\mathrm{p} 22.33$; $\mathrm{p} 11.32) / 45, \mathrm{X} / 45$, and $\operatorname{dic}(\mathrm{X} ; \mathrm{Y})(\mathrm{p} 22.33 ; \mathrm{p} 11.32)$, were associated with ovotesticular $\mathrm{DSD}^{23}$, indicating that gene position/epigenetic effects could be contributed to genital ambiguity. Indeed, histone lysine acetyl-transferases were involved in epigenetic control of the SRY promoter ${ }^{24}$. Ovotesticular development is involved in, at least, two types of cell divisions (mitosis and meiosis) and two types of gametogenesis processes (spermatogenesis and oogenesis) within an ovotestis organ. Together, these data indicated that ovotesticular formation was a very complicated pathological process involved in multiple molecular and cellular events; however, the underlying etiology and molecular mechanisms are largely unknown.

Gonadal dysgenesis and ovotestis phenotype are often prevalent in animals, including dogs, cats, birds, fishes, amphibians, and reptiles ${ }^{25-29}$. In some animals, ovotestis formation is a physiological process, not a pathological condition, for example, in the teleost Monopterus albus, a new model species for evolution, genetics and development ${ }^{26}$. It begins life as a female, but transforms to a male through an intersex stage. The ovotestis model provides an ideal avenue to get insight into how the ovotestis is formed, although the underlying mechanisms have remained elusive since the 1940 's ${ }^{30,31}$. However, singlecell RNA sequencing (scRNA-seq) technology provides a new possibility to decipher the developmental events in the ovotestis at single-cell resolution. It shows a unique advantage in defining cellular identity, and tracing the cell state and fate in a whole organ ${ }^{32-34}$. Here, we performed extensive scRNA-seq characterization of the ovotestis using the 10x Genomics and related analysis applications. We first performed nonlinear dimensionality-reduction analysis, a uniform manifold approximation and projection (UMAP) ${ }^{35}$, on sequenced datasets after quality control, which yielded the first comprehensive single-cell transcriptional cell atlas of major cell types in the ovotestis, including germline, niche, and stem cells. Our study uncovered developmental changes in the lineages of these cell types. We identified common progenitors of germline stem cells with two states, revealing their bipotential nature to give rise to both spermatogonial stem cells (SSC) and female germline stem cells (FGSC), and two developmental trajectories of five subtypes of germline stem cells. Moreover, we revealed that ovotestis infertility was caused by developmental defects in both oogenesis and spermatogenesis processes, through nuclear phase separation of the proteasomes for degradation of female germline cells, and impaired histone-to-protamine replacement in spermatid differentiation. We also delineated signaling pathways in somatic niche cells and their interactive roles with germline cells in determining the cell fates of both testis and ovary differentiation. Together, our results provide a roadmap of germline, niche, and stem cell development in ovotestis, and reveal the molecular and cellular mechanisms underlying ovotestis formation in intersex.

\section{Results \\ Two developmental trajectories of five subtypes of germline stem cells}

Histologically, the ovotestis had atretic and degraded follicles (Fig. 1A, B), and spermatogonia, spermatocytes, and round spermatids (Fig. 1C). To explore the differentiation potential of ovotestis, we first sought to identify cell types in ovotestis using the single-cell RNA sequencing technology. We collected ovotestis samples from three intersex individuals and dissociated them into single cells for scRNA-seq. The sequencing depth was over 47,000 reads for each cell (Table S1), median detected genes were 3126 per cell, and median UMIs (unique molecular identifiers) were 9259 (Table S2). After discarding poor-quality cells, nearly 10,000 cells remained for clustering and typing. To identify cell clusters, we employed a nonlinear dimensionality-reduction technique, UMAP. Together with known marker genes and annotations (Table S3), 13 cell clusters were identified, including germline stem cells, primordial oocytes, spermatogonia, spermatocytes, round spermatids, and somatic niche cells (Sertoli and Leydig cells) (Fig. S1A). UMAP maps showed that each cluster was distinguished from other clusters with marker genes, for example, nanos 2 and nanos3 in germline stem cells, kif20a and kmt5a in spermatogonia B, spo11 and meiob in spermatocytes, izumo1 and spaca 6 in round spermatids, amh and $w t 1 b$ in Sertoli cells, and cyp17a1 and star in Leydig cells (Fig. S1B).

As germline stem cells (GSC) were in cluster 1 (Fig. S1A, B), we next performed re-clustering analysis on the cluster to identify cell subtypes or states, which revealed the existence of five distinct subclusters (Fig. 1D, E). Of these, four subclusters $(1,2,4$, and 5) represented four states of germline stem cells, early-GSC (E-GSC), 


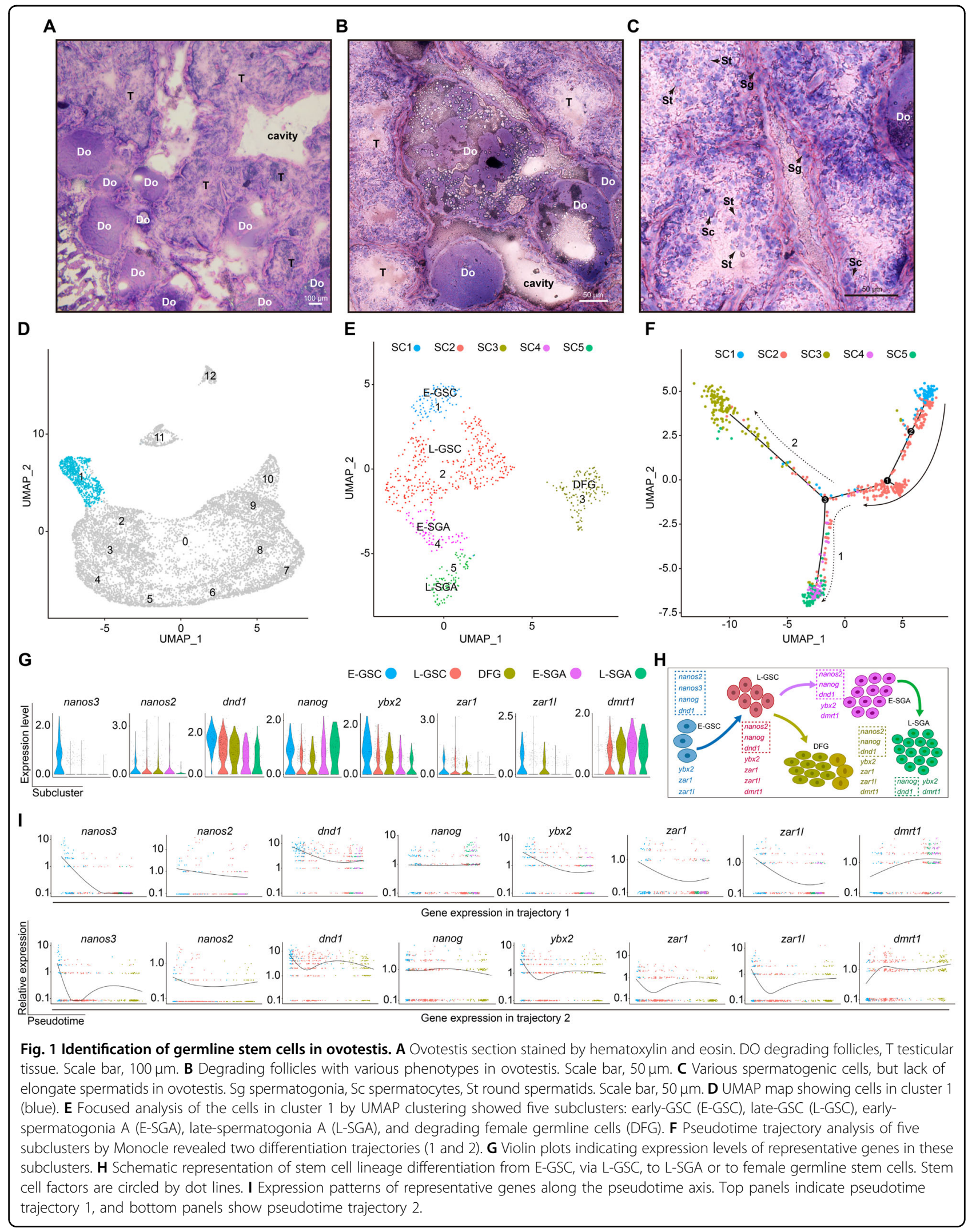


late-GSC (L-GSC), early-spermatogonia A (E-SGA), and late-spermatogonia A (L-SGA), while subcluster 3 was a group of female germline cells including female germline stem cells (FGSC). Pseudotime analysis revealed two developmental trajectories of these five subclusters; trajectory 1 transitioned from L-GSC to L-SGA for spermatogenesis, whereas trajectory 2 showed a distinct fate from L-GSC to female FGSC for oogenesis (Fig. 1F-H). Thus, E-GSC and L-GSC were two differentiation states of common progenitors of germline stem cells. E-GSC cells were primordial germline stem cells and L-GSC cells were bipotential to differentiate into either male (spermatogonia A) or female germline stem cells. Subcluster 3 contains FGSC as indicated by pluripotency markers nanog2, nanog and dnd1, in addition to zar1 and zarll, key genes for oogenesis ${ }^{36}$ (Fig. 1G-I and Fig. S1A, B). These data suggested that L-GSC-to-E-SGA or L-GSC-toFGSC transition potential existed in ovotestis. In line with this fact, upregulated genes were enriched in these two transitioning processes (Fig. S2A).

The expression patterns of key PGC markers ${ }^{37,38}$, including nanos 2 , nanos 3 , and $d n d 1$, along with pluripotency marker nanog in E-GSC, confirmed the pluripotency of E-GSC cells. nanos3 was expressed slightly higher in E-GSC than in L-GSC, indicating the relatively higher stemness of E-GSC in comparison with L-GSC (Fig. 1G-I). Pseudotime trajectory and expression of known cell state markers also indicated that E-SGA and L-SGA were two states of the type A spermatogonia, with high expression of $d m r t 1$, but scarce expression of zar1 and zar1l (Fig. 1F-I). In mammals, Dmrt1 was also expressed in the type A spermatogonia ${ }^{39}$. L-SGA expressed pluripotency markers nanog and $d n d 1$, whereas spermatogonia $B$ in cluster 2 did not express these two markers, but kif20a and kmt5a, important genes for cell division ${ }^{40}$ (Fig. S1B), indicating a transition from L-SGA to spermatogonia $B$ in subsequent development. Notably, genes for DNA synthesis and mitosis were highly expressed in the germline stem cells, in particular, in type A spermatogonia, indicating that these germline stem cells were actively divided (Fig. S2B, C).

\section{Nuclear phase separation of the proteasomes promotes degradation of female germline cells}

Considering functional loss of oogenesis, we explored the female germline cell fate in subcluster 3 (Fig. S3A). Apoptosis is a well-known mechanism for cell death. We indeed detected upregulation of pro-apoptosis gene expression, including gadd45a, bax, and casp $3 a$, in subcluster 3 (Fig. S3B). We further investigated whether autophagy genes are involved in the process. All these genes in autophagy pathway, along with key genes for autophagosome induction (atg13, ulk2, atg5, becn1, atg2b and pi3kr4), elongation (lc3a, lc3b, atg $4 b$, and wipi2), and fusion with lysosome (tecpr1b and epg5), were upregulated in subcluster 3 (Fig. S3C). Moreover, we found that $e s r 2 b$ and $\operatorname{atg} 13$ were upregulated in subcluster 3 simultaneously (Fig. S3D). Functional tests showed that Esr2b binding to the atg13 promoter was essential for activation of atg13 transcription (Fig. S3E-H). Thus, autophagy was involved in female germline cell degradation in subcluster 3 (Fig. S3I).

Importantly, the ubiquitination pathway was enriched in subcluster 3 by KEGG analysis of differential expression genes, and most of the ubiquitination pathway genes were obviously upregulated, including ubiquitinconjugating enzymes ube1, ube2, and ube3 (Fig. 2A). This result suggested an importance of ubiquitinationmediated degradation of the female germ cells, because these enzymes catalyzed ubiquitin conjugation to mark cellular proteins for degradation ${ }^{41}$. To obtain further functional insights, we analyzed expression patterns of key genes involved in the nuclear proteasome foci, and found upregulation of key regulator rad23b, including rad23ba and $\mathrm{rad} 23 \mathrm{bb}$ in subcluster 3 (Fig. 2B). Rad23 regulated proteasome foci formation by phase separation in the nucleoplasm ${ }^{42}$. Under physiological and pathological types of stress, formation of proteasome foci in the nucleus was a new mechanism for proteasome degradation of ribosomal proteins ${ }^{42}$, and loss-of-function mutations in most ribosomal proteins were lethal for cells ${ }^{43}$. Thus, the ubiquitination-dependent proteasome pathway in the nucleus probably mediated degradation of the female germ cells. To verify this, we performed immunofluorescence analysis of Psmb2, a key component of the foci, in ovotestis. Proteasome foci were detected in the germinal cradle (early-stage female germ cells and surrounding supporting cells) in ovotestis, particularly in the nuclei of germ cells and in the cytoplasm of supporting cells (Fig. 2C). Furthermore, proteasome foci were formed in the nuclei of ovary cells $(\mathrm{CHO})$ under hyperosmotic stress (0.2 M sucrose) (Fig. 2D). Notably, overexpression of Rad23ba in CHO cells can promote formation of the foci indicated by endogenous PSMB2, and number of the foci in the nuclei was increased obviously in comparison with the control group (Fig. 2E, F). Considering the essential roles of ribosomal proteins for cell survival, we tested a set of ribosomal proteins and found Rps 26 and Rps2 were co-localized with Psmb2 in the nuclei upon stimulation with $0.2 \mathrm{M}$ sucrose for $30 \mathrm{~min}$ (Fig. $2 \mathrm{G}$ and Fig. S3J), indicating that Rps26 and Rps2 were degraded substrates in the proteasomes. Further time-lapse imaging showed that the proteasome foci with Psmb2 can fuse together, suggesting a nature of liquid droplets of the foci in the living cells (Fig. $2 \mathrm{H}$ and Supplementary Videos 1 and 2). Overall, these data suggested that nuclear proteasomes promoted degradation of female germline cells by ubiquitination-dependent phase separation (Fig. 2I). 

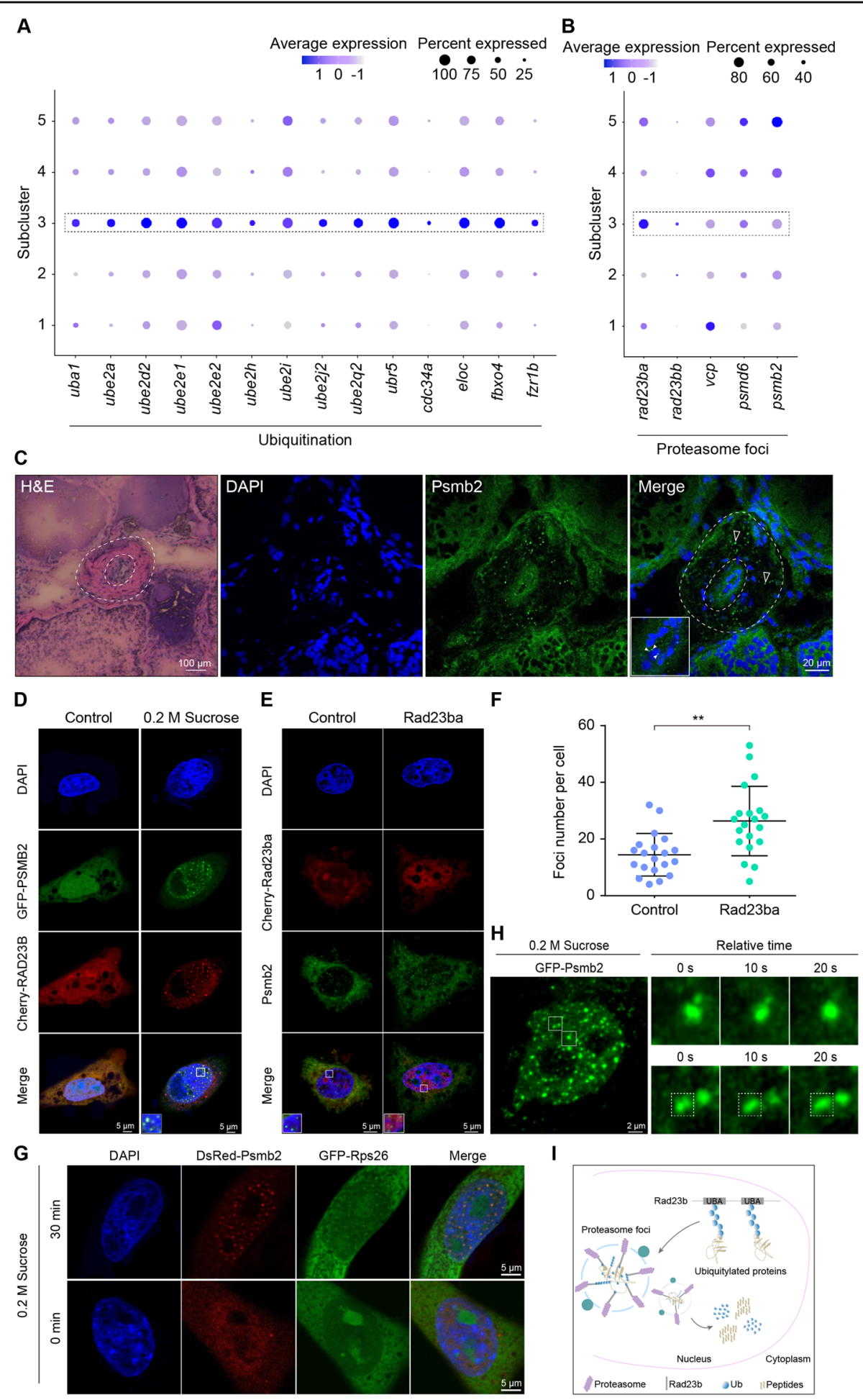

Fig. 2 (See legend on next page.) 
(see figure on previous page)

Fig. 2 Nuclear phase separation of the proteasome for degradation of female germline cells. A Dotplot showing the expression of ubiquitination associated genes among subclusters. B Expression levels of key genes in proteasome foci formation among subclusters. C Immunofluorescence analysis of proteasome foci using Psmb2 antibody in ovotestis (secondary antibody, FITC-conjugated immunoPure goat antirabbit $\lg \mathrm{G}(\mathrm{H}+\mathrm{L})$ ). Proteasome foci were located in the nuclei of germ cells (white solid arrowheads) and in the cytoplasm of supporting cells (white hollow arrowheads). The nuclei were stained with DAPI. The outer dotted circle indicates the degrading region and the inner dotted circle shows the germinal cradle. Scale bar: H\&E, $100 \mu \mathrm{m}$; immunofluorescence, $20 \mu \mathrm{m}$. D Formation of proteasome foci in the nuclei of CHO cells. The cells were treated with $50 \mu \mathrm{M} \mathrm{MG}-132$ for $1 \mathrm{~h}$ and then stimulated with $0.2 \mathrm{M}$ sucrose for $30 \mathrm{~min}$. PSMB2 (green) and RAD23B (red) were colocalized in the nuclei. The nuclei were stained by DAPI. The enlarged images originated from the regions with white square. Scale bar, $5 \mu \mathrm{m}$. E Overexpression of Rad23ba promotes the formation of proteasome foci. Endogenous proteasome foci were detected by PSMB2 antibody in CHO cells. PSMB2 (green) and Rad23ba (red) were colocalized in the nuclei. The nuclei were stained with DAPI. The cells were treated with $50 \mu \mathrm{M} \mathrm{MG-132} \mathrm{for} 1 \mathrm{~h}$ and then stimulated with $0.2 \mathrm{M}$ sucrose for $30 \mathrm{~min}$. The enlarged images originated from the regions with white square. Scale bar, $5 \mu \mathrm{m}$. $\mathbf{F}$ Statistical analysis showing the proteasome foci number per cell. Twenty cells were counted for each group. Data were mean $\pm S D,{ }^{*} P<0.01$. G Co-localization of Psmb2 (red) and Rps26 (green) in nucleus. The cells were stimulated with $0.2 \mathrm{M}$ sucrose for $30 \mathrm{~min}$. The nuclei were stained by DAPI. Scale bar, $5 \mu \mathrm{m}$. H Proteasome foci fusion in living CHO cells expressing GFP-Psmb2. The cells were treated with $0.2 \mathrm{M}$ sucrose. Right panel, enlarged time-course views of the square in the left panel. Scale bar, $2 \mu \mathrm{m}$. I The diagram indicating the nuclear phase separation of proteasome and ubiquitylated protein degradation. The proteasome foci consist of Rad23b, polyubiquitin chain proteins, and proteasome.

\section{Signaling pathways in gonadal niche cells reveal distinct} fate between oogenesis and spermatogenesis

Considering key roles of the gonadal niche for germline cell development, we investigated signaling pathway activities for oogenesis. UMAP analysis showed that marker genes of granulosa cells were scarcely expressed, for example, faint expression of foxl2 was detected in cluster 11 (Fig. 3A). There was also only $10.5 \%$ of Foxl $2^{+}$ cells in the cluster 11 , of which, $\sim 6 \%$ were co-expressed with Sertoli markers, TGF $\beta$ (transforming growth factor beta) signaling pathway genes. Moreover, the foxl2 downstream factor cyp19a1a was also scarcely detected (Fig. 3B), indicating loss of biosynthesis pathway of female sex hormone in the ovotestis (Fig. 3C). Hedgehog signaling pathway was essential for follicle development ${ }^{44}$. Key genes in the hedgehog signaling pathway in theca cells, including ptch1/2, smo, hhip, gli1, gli2, and gli3, were scarcely detected (Fig. 3D), indicating loss of hedgehog signaling pathway in ovotestis (Fig. 3E). In addition, expression of nid1, a basal lamina-specific adhesive protein in theca cells, was also very low (Fig. 3D), which was important for production of basal membranes of follicles $^{45}$. Nevertheless, immunofluorescence analysis showed that theca cells can be detected by Krt8, which was expressed in cluster12 (Fig. 3D, F). However, Krt8 was not expressed in granulosa cells in the degraded follicles (Fig. 3F). These data suggested that the gonadal niche for oogenesis was functionally lost, although granulosa cells and theca cells co-existed in ovotestis.

As gonadal niche is essential for spermatogenesis, we then addressed signaling pathway activities in Sertoli cells and Leydig cells in ovotestis. KEGG pathway analysis by g: profiler $^{46}$ showed that TGF $\beta$ family genes were enriched in Sertoli cells, including $a m h, b m p 4, s m a d 7, d c n$, and $i d 1$. UMAP plots were built for Sertoli cells integrated by expression levels of TGF $\beta$ pathway genes (Fig. 4A, B). Key factors, including Amh, Bmp4, Dcn, Smad7, and their target Id1, in the pathway, were active in Sertoli cell cluster (Fig. 4C), suggesting functional Sertoli cells in ovotestis, which were consistent with the fact that Amh signaling is required for male development ${ }^{47}$. Further GO term enrichment showed that steroidogenesis process was enriched in Leydig cells. UMAP analysis of steroid biosynthesis indicated that male steroid biosynthesis factors, including star/starl, cyp11a1, cyp17a1, and hsd3b1, were highly expressed in Leydig cell cluster (Fig. 4D, E), which were key proteins for testosterone synthesis, In particular, Star and Starl were responsible for rate-limiting transport of cholesterol into the mitochondria for further steroidogenesis $^{48}$, Cyp11a1, Cyp17a1, and Hsd3b1 worked in their downstream in the pathway (Fig. 4F). In addition, UMAP and immunofluorescence analysis showed that Sertoli and Leydig cells can be detected by Krt8, a protein for formation of intermediate filaments (Fig. 4G, H), and Sertoli and Leydig cells can also be detected by Cldn11, a tight junction protein (Fig. 4I, J). These results suggested that both Sertoli cells and Leydig cells were functional in ovotestis.

\section{Spermatogenesis arrests at round spermatid phase owing lack of transition from histone to protamine}

Phenotypically, round spermatids, but not elongated spermatids, were observed in ovotestis (Fig. 1C). Interestingly, two states (early and late) of round spermatids were detected (Fig. 5A). Both the number of expressed genes and the number of UMIs in the late stage of round spermatids were the most abundant among all clusters (Fig. S1C, D), suggesting active gene expressions in the late stage of round spermatids in ovotestis. To better understand the mechanisms of spermatogenesis arrest in the late round spermatids in ovotestis, developmental trajectory was used to track developmental order of the cell populations among clusters. This analysis indicated that developmental trajectory from cluster 2 to 10 


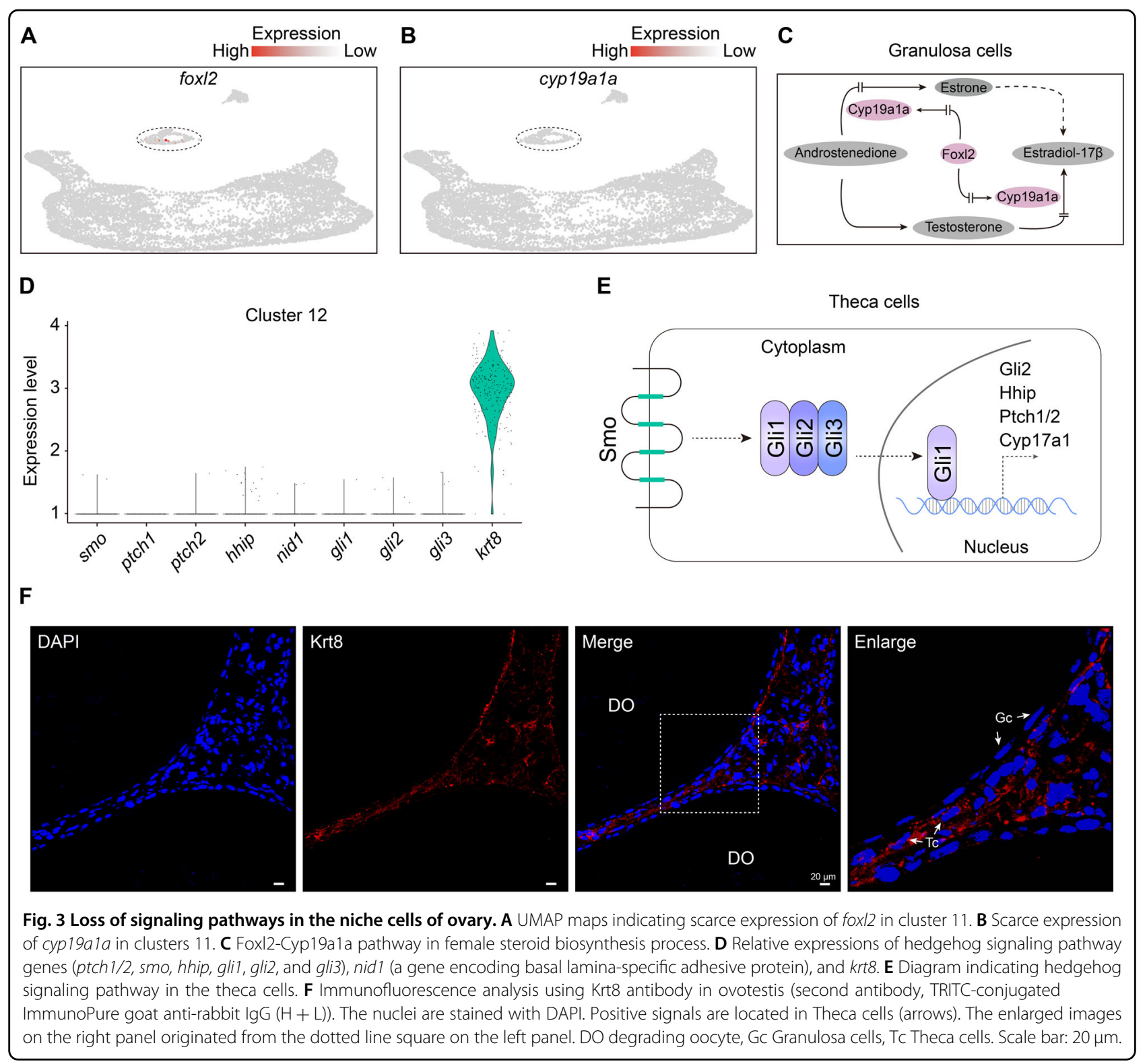

represented meiosis process from spermatogonia $\mathrm{B}$, spermatocytes, to late stage of round spermatids (Fig. 5A). Focused analysis of primary spermatocytes reveals developmental progression of the meiosis I, in which four subclusters, premeiotic $\mathrm{S}$ phase, leptotene/zygotene, pachytene, and metaphase/anaphase, were detected (Fig. S4A, B). Pseudotime trajectory with corresponding marker genes based on DEGs of each subcluster confirmed the developmental process of primary spermatocytes (Fig. S4C-G).

Gene ontology (GO) of both upregulated and downregulated genes among clusters during spermatogenesis showed functional changes with feature genes of high expression from spermatogonia $\mathrm{B}$ to round spermatids (Fig. 5B, C). Immunofluorescence analysis indicated expression of Sycp3, a marker of synaptonemal complex in spermatocytes, and Spag6, a component of the central apparatus, in round spermatids, suggesting normal development in spermatocytes and round spermatids (Fig. 5D, E). These analyses indicated that spermatogenesis processes from spermatogonia $\mathrm{B}$, spermatocytes, to early stage of round spermatids proceeded properly. To get insight into spermiogenesis arrests, we performed expression analysis by UMAP mapping. This analysis indicated that representative genes of round spermatids, including spag6, izumo1 and spaca6, were expressed in both cluster 9 and 10. In contrast, protamine was scarcely detected in both stages of round spermatids and even not in the whole ovotestis, but in testis (Fig. 5F, G). However, four types of histone genes, including $h 2 a, h 2 b, h 3$, and 


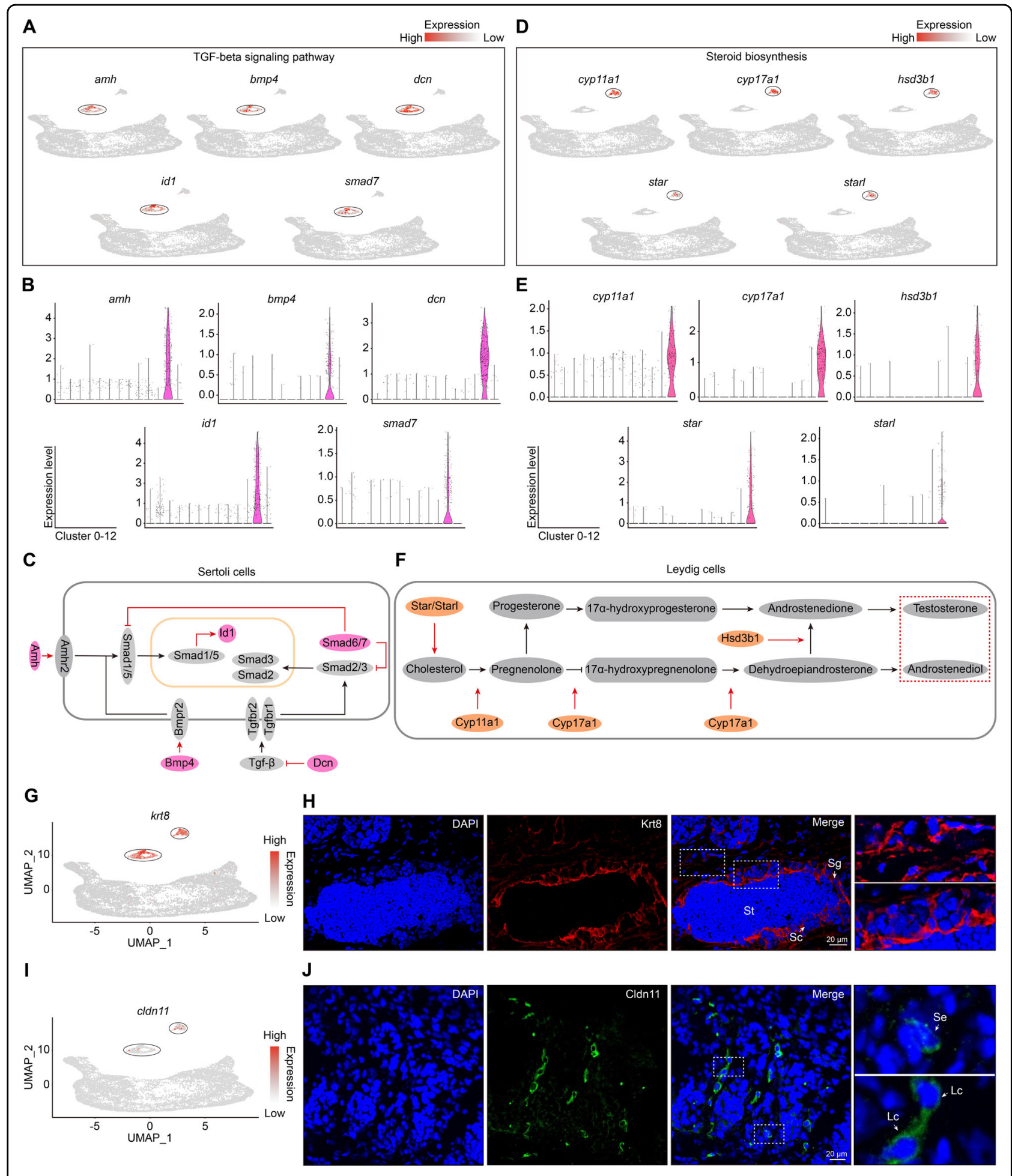

Fig. 4 (See legend on next page.) 
(see figure on previous page)

Fig. 4 Signaling pathways in the niche cells of testis. A Key genes and expression patterns in TGF $\beta$ signaling pathway in Sertoli cells in cluster 11. Cluster-specific expression is indicated by black circles. B Violin plots showing expression levels of TGF $\beta$ signaling pathway genes. C TGF $\beta$ signaling pathway in Sertoli cells, highlighting the key genes in red ovals. D Key genes and expression patterns in steroid biosynthesis process in cluster 12. Cluster-specific expression is shown in black circles. $\mathbf{E}$ Violin plots showing expression levels of steroid biosynthesis genes. $\mathbf{F}$ Male steroid biosynthesis process in Leydig cells, highlighting the key genes in orange ovals. G UMAP map of krt8 showing expression in both cluster 11 and 12 , indicated by black circles. $\mathbf{H}$ Immunofluorescence analysis using Krt8 antibody in ovotestis (second antibody, TRITC-conjugated immunoPure goat anti-rabbit IgG $(\mathrm{H}+\mathrm{L})$ ). The nuclei were stained with DAPI. Positive signals were located in Sertoli and Leydig cells (arrows). The enlarged images on the right panel originated from the dotted squares on the left panel. Sg spermatogonia, Sc spermatocytes, St round spermatids, Lc Leydig cells, Se Sertoli cells. Scale bar: $20 \mu \mathrm{m}$. I UMAP map of cldn 11 showing expression in both cluster 11 and 12, indicated by black circles. $\mathbf{J}$ Immunofluorescence analysis using Cldn11 antibody in ovotestis (second antibody, FITC-conjugated immunoPure goat anti-rabbit $\lg G(\mathrm{H}+\mathrm{L})$ ). The nuclei were stained with DAPI. Positive signals were located in Sertoli and Leydig cells (arrows). The enlarged images on the right panel originated from the dotted squares on the left panel. Lc Leydig cells, Se Sertoli cells. Scale bar: $20 \mu \mathrm{m}$.

h4, were expressed (Fig. 5F), and further immunofluorescence of $\mathrm{H} 2 \mathrm{~b}$ protein confirmed its expression in both stages of round spermatids in ovotestis (Fig. $5 \mathrm{H}$ ), suggesting a histone retention in round spermatids in ovotestis. In contrast, in males, histone $\mathrm{H} 2 \mathrm{~b}$ was not observed in both round and elongate spermatids, suggesting that histones had been replaced by protamine in testis (Fig. 5I). Histone-to-protamine replacement is essential for producing functional sperm cells, and lack of histone replacement by protamine during post-meiotic transition of round spermatid to elongate spermatids led to male infertility ${ }^{49}$. Considering an enrichment of early round spermatids and a dramatic decline of late round spermatids (Fig. 5J), spermatogenesis would be arrested at early phase of round spermatids owing to lack of transition from histone to protamine (Fig. 5K). Overall, these data suggested that histone-to-protamine replacement cannot be performed due to deficiency of protamine expression, which thus led to developmental defects of elongate spermatids in ovotestis.

\section{Discussion}

Infertile intersex often appears in individuals under certain pathological and physiological conditions, in which the gonad consists of both testis and ovary tissues, that is ovotestis. Patients who suffer from ovotestis are infertile and have many health problems ${ }^{1}$. However, how the ovotestis is formed remains elusive. Here, our study provides an overview of cell identities and a roadmap of germline, niche, and stem cell development in ovotestis, and uncovers the molecular and cellular mechanisms underlying ovotestis formation.

We found a common progenitor of germline stem cells with two developmental states, E-GSC and L-GSC, in ovotestis, which have a bipotential nature to differentiate into both spermatogonial stem cells and female germline stem cells. The developmental trajectories of five subtypes of germline stem cells confirmed the fate of differentiation to either oogenesis or spermatogenesis. Thus, E-GSC and L-GSC are similar to primordial germ cells (PGCs) in the developing embryonic gonad. The fact that they express a group of PGC markers, including nanos2, nanos3, nanog, and $d n d 1$, also supports this viewpoint. Human PGCs are induced in the epiblast in early embryos and migrate to colonize the genital ridge in both $\operatorname{sexes}^{50}$. In male embryos, these PGCs can differentiate into spermatogonial stem cells and relevant germline cells, whereas in female embryos, they will develop into female germline stem cells and female germline cells ${ }^{51}$. In human adults, PGCs were not detected, and only PGC-like cells were observed in neonatal testes ${ }^{52}$. The findings of the common progenitors of germline stem cells, the E-GSC and L-GSC, in ovotestis, suggested that ovotestis possesses a developmental bipotential, attributed to the common stem cell progenitors. Our discovery of the common germline stem cell progenitors enriches our understanding of stem cell development.

Importantly, we uncovered developmental changes in the germline and niche cell lineages, and the molecular and cellular mechanisms underlying ovotestis formation. The mechanisms are involved in two disrupted processes of coordinating action. Female germline cells are degraded by nuclear phase separation of the proteasomes, and histone-to-protamine replacement is impaired in spermatid differentiation, which lead to developmental defects in both oogenesis and spermatogenesis processes in the ovotestis. On the one side, histone replacement by protamine is an essential process for chromatin remodeling in spermatid differentiation. Protamine mutations or aberrant histone retention results in failure of histone-toprotamine replacement, thus causing male infertility in humans and mice ${ }^{49,53}$. Our study reveals that deficiency of protamine expression is a major cause of infertility owing to lack of histone to protamine exchange in the round spermatid differentiation in ovotestis. On the other side, we found that nuclear phase separation of the proteasomes is a robust process in female germline cells, which is a degradation mechanism of ubiquitination-dependent proteasomes in the nuclei. Ubiquitination-mediated proteasome foci in the nucleoplasm were recently found in 

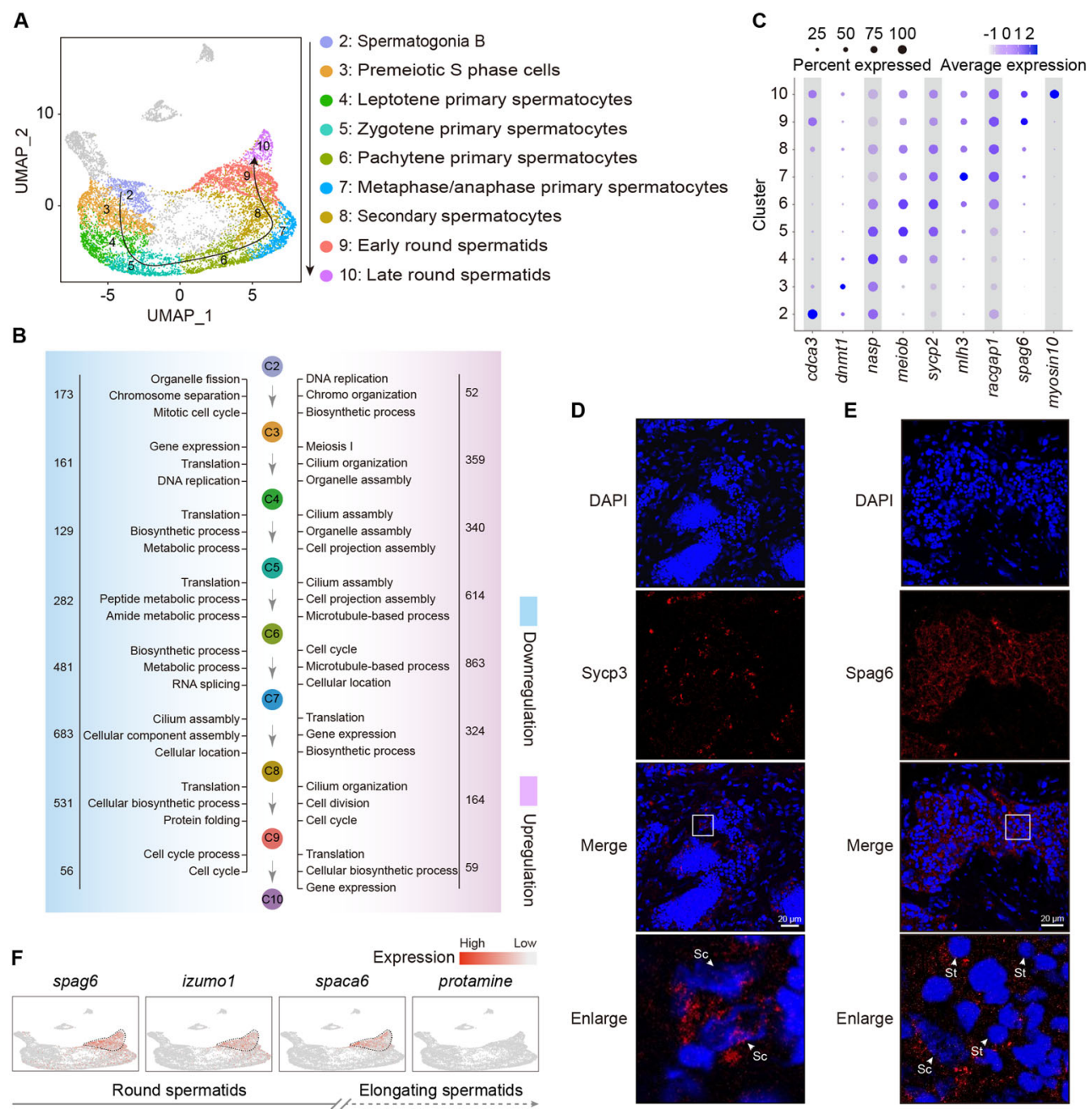

E
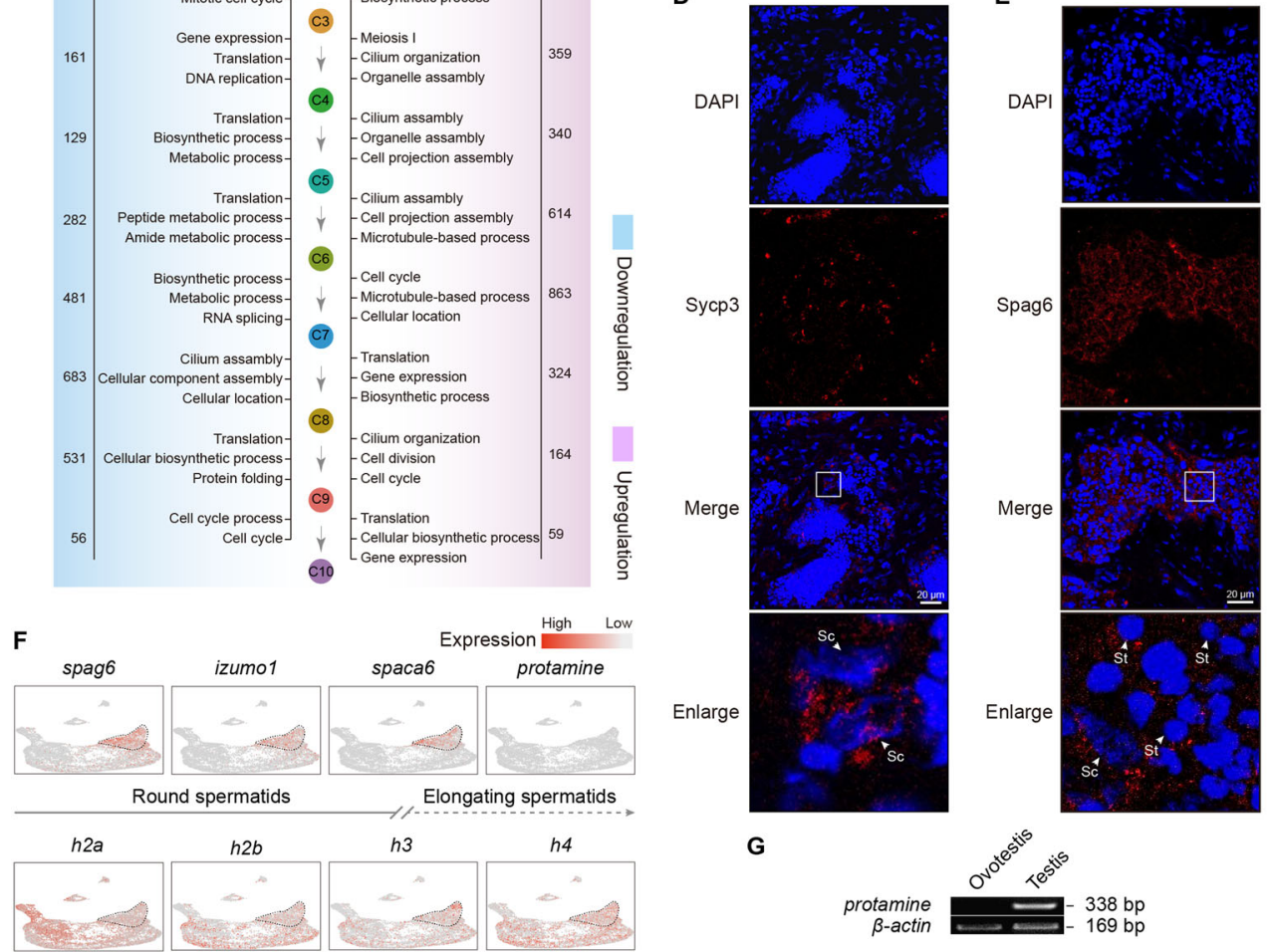

J

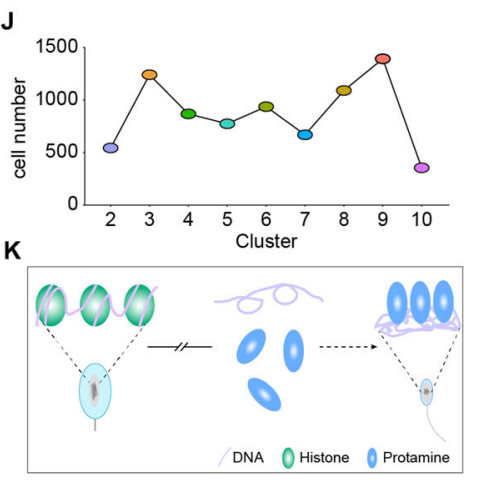

H DAPI
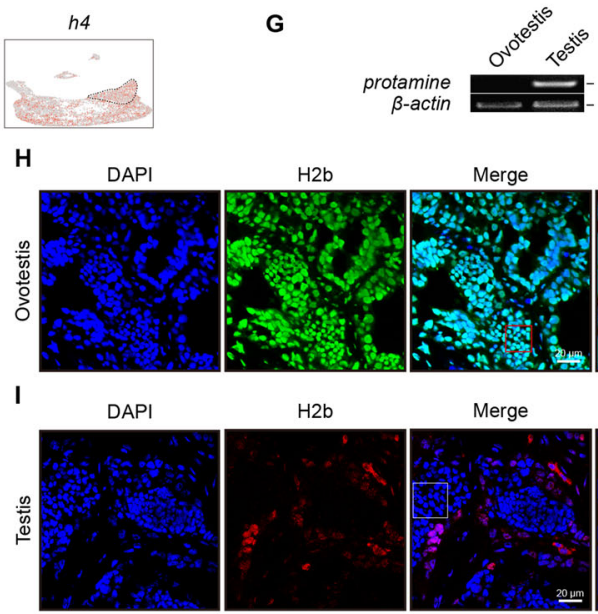

$338 \mathrm{bp}$
$169 \mathrm{bp}$

DAPI
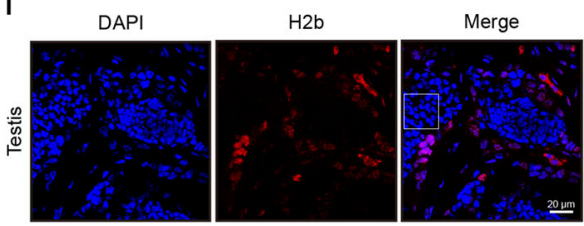

Enlarge

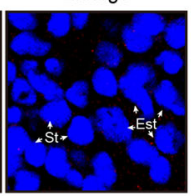

Fig. 5 (See legend on next page.) 
(see figure on previous page)

Fig. 5 Spermatogenesis arrests at round spermatid phase owing to lack of transition from histones to protamine. A Developmental trajectory from spermatogonia B to round spermatids. B Representative gene ontology (GO) terms of upregulated and downregulated genes among clusters during spermatogenesis. Downregulated GO terms are in blue, upregulated GO terms are in pink. The number of upregulated and downregulated genes is indicated. C Dotplot showing the expression of representative genes in each cluster. D Immunofluorescence analysis of ovotestis using Sycp3 antibody (second antibody, TRITC-conjugated immunopure goat anti-rabbit $\lg G(\mathrm{H}+\mathrm{L})$ ). The nuclei were stained with DAPI. Sc spermatocytes. Scale bar: $20 \mu \mathrm{m}$. E Immunofluorescence analysis of ovotestis using Spag6 antibody (second antibody, TRITC-conjugated immunopure goat anti-rabbit $\lg G(H+L))$. The nuclei were stained with DAPI. Sc spermatocytes, St round spermatids. Scale bar: 20 Mm. F UMAP maps showing expression patterns of marker genes during spermatogenesis. Protamine was scarcely expressed, while histones were obviously expressed in round spermatids. G RT-PCR analysis of protamine expression pattern in ovotestis and testis. RT-PCR was performed using CDNAs as template. $\beta$-actin was used as an internal control. $\mathbf{H}$ Immunofluorescence analysis of ovotestis using H2b antibody (second antibody, FITC-conjugated immunopure goat anti-rabbit $\lg \mathrm{G}(\mathrm{H}+\mathrm{L})$ ). The nuclei were stained with DAPI. St, round spermatids. Scale bar: $20 \mu \mathrm{m}$. I Immunofluorescence analysis of testis using $\mathrm{H} 2 \mathrm{~b}$ antibody (second antibody, TRITC-conjugated immunopure goat anti-rabbit $\lg \mathrm{G}(\mathrm{H}+\mathrm{L}$ )). The nuclei were stained with DAPI. Round spermatids (St) and elongate spermatids (Est) are indicated by arrows. Scale bar: $20 \mu \mathrm{m}$. J Statistical analysis of cell number showing differentiation arrest from early round spermatids (cluster 9) to late round spermatids (cluster 10). $\mathbf{K}$ Schematic model of differentiation arrest from early round spermatids to late round spermatids owing to lack of transition from histone to protamine.

mammalian cells under various types of stress, including mouse stem cells, which can degrade key components in the nucleus, such as ribosomal proteins ${ }^{42}$. Consistent with this, loss-of-function mutations in most ribosomal proteins were lethal ${ }^{43}$. In addition, degradation of female germline cells is probably induced by aberrant signaling of the gonadal niche cells, owing to functional loss of the gonadal niche for oogenesis in the ovotestis. Thus, we suggest that the female germline cell degradation could be mediated by the nuclear proteasome pathway, together with deficiency signaling of the gonadal niche in ovotestis. Overall, our results provide a roadmap of germline, niche, and stem cell development in ovotestis and the corresponding molecular and cellular mechanisms underlying infertility.

\section{Materials and methods}

\section{Experimental animals}

The teleost fish Monopterus albus individuals were obtained from fish farms in Wuhan, Hubei Province, China. Adult animals (male, intersex) were used without randomization. All animal experiments and methods were performed in accordance with the relevant approved guidelines and regulations, as well as under the approval of the Ethics Committee of Wuhan University.

\section{Antibodies}

Primary antibodies: Anti-Spag6 (A3088), Anti-Krt8 (A1024), Anti-Psmb2 (A5483), Anti-Cldn11 (A2593) were purchased from ABclonal, Wuhan, China. Anti-H2b (ET1612-25) was purchased from Huaan Biotechnology, Hangzhou, China. Anti-Sycp3 (ab97672) was purchased from Abcam, Cambridge, UK. Fluorescent antibodies: TRITC-conjugated ImmunoPure goat anti-rabbit IgG $(\mathrm{H}+\mathrm{L})(\mathrm{Cat} \# \mathrm{ZF}-0316)$ and FITC-conjugated ImmunoPure goat anti-rabbit $\operatorname{IgG}(\mathrm{H}+\mathrm{L})$ (Cat\# ZF-0311) were purchased from Feiyi Technology, Wuhan, China.

\section{Plasmid constructs}

Full-length esr2b (XM_020611557.1) was cloned into pcDNA3.0 using EcoRI and SalI to generate pCMV$e s r 2 b$. Three deletion fragments of the atg13 (XM_020600753.1) promoter were amplified from genomic DNA, using KpnI and XhoI to generate the pGL3-basic vector (E1751, Promega, Madison, WI, USA). Full-length rad23ba (XM_020600944.1) was cloned into pSico-Cherry-FLAG using EcoRI and XhoI to generate Cherry-FLAG-rad23ba. Full-length Rad23b (NM_009011.4) was cloned into pSico-Cherry-FLAG using EcoRI and XhoI to generate Cherry-FLAGRad23b. Full-length Psmb2 (NM_011970.4) and psmb2 (XM_020597689.1) were cloned into pEGFP-N1 using XhoI and EcoRI to generate GFP-Psmb2 and GFPpsmb2. The primers and PCR conditions are described in Table S4. Site-directed mutagenesis for the Esr2b binding sites was performed using the primers described in Table S4. All constructs were confirmed by sequencing (TSINGKE, Beijing, China).

\section{Cell culture and transfection}

HEK293T cells (3142C0001000001715) and CHO cells (GDC0018) were obtained from China Center for Type Culture Collection. HEK293T cells were cultured in high glucose Dulbecco's modified Eagle's medium (DMEM) (SH30022.01B, HyClone, Logan, USA) with 12\% fetal bovine serum (FBS) (P30-330250, PAN-Biotech, Aidenbach, Germany) and CHO cells were cultured in DMEM/ F12 with $10 \%$ FBS. All cells were cultured at $37^{\circ} \mathrm{C}$ in a $5 \%$ $\mathrm{CO}_{2}$ cell incubator. LipofectamineTM 2000 (11668027, Invitrogen, Shanghai, China) was used for transfection according to the routine protocol. For hyperosmotic stress, CHO cells were treated with $50 \mu \mathrm{M}$ MG-132 (HY13259, MCE, Shanghai, China) for $1 \mathrm{~h}$, and then were stimulated using $0.2 \mathrm{M}$ sucrose for $30 \mathrm{~min}$ (S818049, MACLIN, Shanghai, China). 


\section{Immunofluorescence and living cell analysis}

Immunofluorescence analysis was performed as described previously ${ }^{54}$. Briefly, $\mathrm{CHO}$ cells were cultured on glass coverslips, and gonadal tissues were embedded in OCT medium (4583, Tissue-Tek, Miles, USA) and cut into a series of $7 \mu \mathrm{m}$ sections using a cryostat (CM1850, Leica, Germany). All samples were fixed with $4 \%$ paraformaldehyde for $20 \mathrm{~min}$ at room temperature and permeabilized with 1\% Triton X-100 (9002-93-1, Sigma-Aldrich, USA) in PBS for $10 \mathrm{~min}$ and then blocked in 5\% BSA for $30 \mathrm{~min}$ at room temperature. The sections were incubated with primary antibody in $5 \%$ BSA overnight at $4{ }^{\circ} \mathrm{C}$. After being washed three times with PBS, the samples were subjected to the appropriate fluorescein-conjugated secondary antibody at $37^{\circ} \mathrm{C}$ for $3 \mathrm{~h}$. The nuclei were stained by DAPI (1155MG010, BioFroxx, Germany). Images were captured by confocal fluorescence microscopy (SP8, Leica, Germany). For time-lapse experiments, $\mathrm{CHO}$ cells were initially plated in $35 \mathrm{~mm}$ glass-bottomed dishes (BS-20-GJM, Biosharp, Hefei, China). The cells were transfected with pEGFP-psmb2 plasmid when the cell density reached $50-60 \% .4 \mathrm{~h}$ after transfection, the medium was replaced by fresh medium. After $24 \mathrm{~h}$, the medium was discarded, cells were washed using PBS for one time, treated with $0.2 \mathrm{M}$ sucrose for $30 \mathrm{~min}$, and imaged for 1-2 h. Finally, images were captured by Zeiss confocal fluorescence microscopy (LSM880, Zeiss, Oberkochen, Germany) with an incubator (humidified environment at $37{ }^{\circ} \mathrm{C}$ under $5 \% \mathrm{CO}_{2}$ ). The data were processed by ZEN software (Zeiss, Oberkochen).

\section{Dual-luciferase reporter assays}

For luciferase assays, $293 \mathrm{~T}$ cells per well (48 well plate) were transfected with $0.5 \mu \mathrm{g}$ recombinant constructs and $1 \mathrm{ng}$ pRL-TK (E2241, Promega). Then luciferase activities were measured by a dual-luciferase reporter assay system (E1980, Promega) and a Modulus Single Tube Multimode Reader (Turner Biosystems, Sunnyvale, CA, USA) according to the manufacturer's protocol. The experiments were repeated at least three times, and the results were expressed as the means $\pm \mathrm{SD}$.

\section{Semiquantitative RT-PCR}

TRIzol (R401-01-AA, Vazyme, Nanjing, China) was used to isolate total RNA, which was reverse transcribed using a poly $(\mathrm{T}) 18$ primer and MMLV reverse transcriptase (M1701, Promega). Semiquantitative RT-PCR was used to amplify cDNAs from ovotestis and testis tissues. $\beta$-actin was used as an internal control. The primer sequences are described in Table S4.

\section{Ovotestis sample preparation for single-cell RNA sequencing}

Gonads were collected and washed twice in 1xPBS. Sex was verified by histological analysis of the gonad sections.
The sections were stained by hematoxylin and eosin and images were captured using a Leica microsystem (Leica). Three samples were minced with sterilized scissors after washing three times with D-Hank's, then were digested in collagenase type IV (C5138, Sigma-Aldrich, St Louis, USA) of $1 \mathrm{mg} / \mathrm{ml}$ at $28^{\circ} \mathrm{C}$ for $20 \mathrm{~min}$, with shaking up and down once in every $3 \mathrm{~min}$. The tubes were spun at $300 \mathrm{~g}$ and the supernatant was removed. The samples were washed three times with D-Hank's, then digested with 0.25\% trypsin (1541894, gibco, Grand Island, NY, USA) and Deoxyribonuclease I (EN0521, Thermo Scientific, Rockford, IL, USA) buffered by Leiboviz's L-15 (SH30525.01, HyClone, Utah, USA) medium for $10 \mathrm{~min}$, with shaking up and down once in every $2 \mathrm{~min}$. The digestion was stopped with Leiboviz's L-15 medium supplemented with 10\% FBS (P30-330250, PAN-Biotech, Aidenbach, Germany). After filtering by strainers with mesh sizes of $70 \mu \mathrm{m}$ (15-1070, Biologix, Jinan, Shandong Province, China) and $40 \mu \mathrm{m}$ (15-1040, Biologix), single cells were obtained. The cells were pelleted by centrifugation at $300 \mathrm{~g}$ for $5 \mathrm{~min}$ and resuspended with Leiboviz's L-15 medium supplemented with $10 \%$ FBS. The cell viability was evaluated by trypan blue (T6146, Sigma-Aldrich) staining with a hemocytometer.

\section{Library preparation and sequencing}

Chromium single-cell $3^{\prime}$ reagent kits V3 (10X Genomics, Pleasanton, CA, USA) were used for scRNA-seq library preparation according to the manufacturer's protocols. A single-cell suspension was loaded into the Chromium Controller (10X Genomics) to form single cell GEMs (Gel Bead-in-Emulsions), which were droplets including a bead with cell barcode and a cell in every droplet. RT-PCR reaction was performed in the individual GEM and synthetic cDNA with a specific cell barcode was mixed for amplification; then the cDNA library was prepared based on the manufacturer's instructions (10X Genomics). Sequencing was performed on a BGISEQ-500 (BGI, Shenzhen, China).

\section{Mapping and processing of single-cell RNA-seq data}

Raw sequencing data were demultiplexed using the mkfastq application of CellRanger (CellRanger v 3.1.0) with default parameters to get Fastq files. Reads were aligned to reference genome (M_albus_1.0) using STAR ${ }^{55}$, and barcode and UMI counting were performed to generate feature-barcode matrices by CellRanger count application (Cell Ranger v 3.1.0) with default settings. The feature-barcode matrices were used for further analysis.

Using Seurat package (v3.1.0) ${ }^{56}$ with default parameters, we checked the single cell data to filter cells of good quality. The cells were retained when the expressed gene number of cells was more than 200 and the maximum expressed gene number was less than $90 \%$, with less than 
$5 \%$ of mitochondrial gene expression. We normalized gene expression values relative to the total number of reads in the cell, and the resulting expression values were then multiplied and log-transformed. Subsequently, the most highly variable genes of 2000 were used for further clustering analysis. Nonlinear dimensionality reduction was performed by principal component analysis and a graph-based clustering approach was used for cell clustering. Then, two-dimensional visualization of clusters was generated by uniform manifold approximation and projection (UMAP), which is a nonlinear dimensionalityreduction technique ${ }^{35}$. For each cluster, differentially expressed genes were identified by FindConservedMarkers application (logfc. threshold $>0.25$ and minPct $>0.25$ ) of Seurat. Cell types were identified and annotated manually based on the previously known markers and the cluster specific genes. Pseudotime trajectory analysis was performed by Monocle (v2.10.1) ${ }^{57}$ with default settings based on Seurat clustering data sets.

\section{Gene functional annotation}

GO term and KEGG pathway enrichment analysis of DEGs were performed with online software g:Profiler ${ }^{46}$, which supported statistical analysis and visualization. Representatively significant biology processes and KEGG pathways were selected for further studies, and enrichments with $P$-adjusted $<0.05$ were considered as significant. Open reading frames of DEGs were recognized by GETORF (EMBOSS: 6.5.7.0) ${ }^{58}$, then the ORFs were aligned to the AnimalTFDB database ${ }^{59}$ to annotate transcription factors of DEGs using DIAMOND (v0.8.31) ${ }^{60}$. Transcription factor binding sites were predicted using JASPAR online software ${ }^{61} . P$-adjusted of all used DEGs were less than 0.05 .

\section{Acknowledgements}

The authors thank BGI Shenzhen for single cell sequencing. This work was supported by the National Natural Science Foundation of China (31771487, 31771370, and 31970539).

\section{Author contributions}

Conceptualization, H.C. and R.Z; methodology, X.W.; formal analysis, X.W.; investigation, X.W., F.L., D.S., Y.C., and T. L.; writing-original draft, X.W.; writing-review \& editing, X.W. and R.Z.; supervision, H.C. and R.Z; project administration, H.C. and R.Z.

\section{Funding}

This work was supported by the National Natural Science Foundation of China (31771487, 53131771370, and 31970539).

\section{Data availability}

The accession number for the sequence reported in this paper is GEO: GSE153961.

\section{Ethics statement}

All animal experiments and methods were performed in accordance with the relevant approved guidelines and regulations, as well as under the approval of the Ethics Committee of Wuhan University.
Conflict of interest

The authors declare no competing interests.

\section{Publisher's note}

Springer Nature remains neutral with regard to jurisdictional claims in published maps and institutional affiliations.

Supplementary information The online version contains supplementary material available at https://doi.org/10.1038/s41419-021-03676-x.

Received: 1 March 2021 Revised: 25 March 2021 Accepted: 25 March 2021 Published online: 12 April 2021

\section{References}

1. Hughes, I. A., Houk, C., Ahmed, S. F. \& Lee, P. A. Consensus statement on management of intersex disorders. Arch. Dis. Child. 91, 554-563 (2006).

2. Witchel, S. F. Disorders of sex development. Best. Pract. Res. Clin. Obstet. Gynaecol. 48, 90-102 (2018).

3. Bashamboo, A. \& McElreavey, K. Human sex-determination and disorders of sex-development (DSD). Semin. Cell Dev. Biol. 45, 77-83 (2015).

4. Koopman, P., Gubbay, J., Vivian, N., Goodfellow, P. \& Lovell-Badge, R. Male development of chromosomally female mice transgenic for Sry. Nature 351, 117-121 (1991).

5. Croft, B. et al. Human sex reversal is caused by duplication or deletion of core enhancers upstream of SOX9. Nat. Commun. 9, 5319 (2018).

6. Gonen, N. et al. Sex reversal following deletion of a single distal enhancer of Sox9. Science 360, 1469-1473 (2018).

7. Tomizuka, $K$. et al. R-spondin1 plays an essential role in ovarian development through positively regulating Wnt-4 signaling. Hum. Mol. Genet. 17, 1278-1291 (2008).

8. Cheng, Y. et al. Whole genome-wide chromosome fusion and new gene birth in the Monopterus albus genome. Cell Biosci. 10, 67 (2020).

9. McElreavy, K. et al. XY sex reversal associated with a deletion $5^{\prime}$ to the SRY "HMG box" in the testis-determining region. Proc. Natl Acad. Sci. USA 89, 11016-11020 (1992)

10. McElreavey, K. et al. Loss of sequences $3^{\prime}$ to the testis-determining gene, SRY, including the $Y$ pseudoautosomal boundary associated with partial testicular determination. Proc. Natl Acad. Sci. USA 93, 8590-8594 (1996).

11. Fan, W. et al. A novel missense mutation 224G $>T$ (R75M) in SRY coding region interferes with nuclear import and results in 46, XY complete gonadal dysgenesis. PLOS ONE 11, e0168484 (2016).

12. Helszer, Z. et al. A novel mutation (c. $341 \mathrm{~A}>\mathrm{G}$ ) in the SRY gene in a $46, \mathrm{XY}$ female patient with gonadal dysgenesis. Gene 526, 467-470 (2013).

13. Eozenou, C. et al. Testis formation in $X X$ individuals resulting from novel pathogenic variants in Wilms' tumor 1 (WT1) gene. Proc. Natl Acad. Sci. USA 117. 13680-13688 (2020).

14. Baetens, D. et al. NR5A1 is a novel disease gene for $46, X X$ testicular and ovotesticular disorders of sex development. Genet. Med. 19, 367-376 (2017).

15. Bastian, C. et al. Genetic mutations and somatic anomalies in association with 46,XY gonadal dysgenesis. Fertil. Steril. 103, 1297-1304 (2015).

16. Xue, M. et al. Novel pathogenic mutations in disorders of sex development associated genes cause 46,XY complete gonadal dysgenesis. Gene 718, 144072 (2019).

17. Carvalheira, G. et al. The natural history of a man with ovotesticular $46, X X$ DSD caused by a novel 3-Mb 15q26.2 deletion containing NR2F2 gene. J. Endocr. Soc. 3, 2107-2113 (2019).

18. Bashamboo, A. et al. Loss of function of the nuclear receptor NR2F2, encoding COUP-TF2, causes testis development and cardiac defects in $46, X X$ children. Am. J. Hum. Genet. 102, 487-493 (2018).

19. Mengen, E., Kayhan, G., Kocaay, P. \& Uçaktürk, S. A. A duplication upstream of SOX9 associated with SRY negative 46,XX ovotesticular disorder of sex development: a case report. J. Clin. Res. Pediatr. Endocrinol. 12, 308-314 (2019).

20. Vetro, A. et al. Testis development in the absence of SRY: chromosomal rearrangements at SOX9 and SOX3. Eur. J. Hum. Genet. 23, 1025-1032 (2015).

21. Grinspon, R. P. et al. $46, X X$ ovotesticular DSD associated with a SOX3 gene duplication in a SRY-negative boy. Clin. Endocrinol. 85, 673-675 (2016). 
22. van der Zwan, Y. G., Biermann, K., Wolffenbuttel, K. P., Cools, M. \& Looijenga, L. H. Gonadal maldevelopment as risk factor for germ cell cancer: towards a clinical decision model. Eur. Urol. 67, 692-701 (2015).

23. Mazen, I. M. et al. Unique karyotype: $\operatorname{mos} 46, X, \operatorname{dic}(X ; Y)(p 22.33 ; p 11.32) / 45, X / 45$, $\operatorname{dic}(X ; Y)(p 22.33 ; p 11.32)$ in an Egyptian patient with Ovotesticular disorder of sexual development. Sex. Dev. 7, 235-243 (2013)

24. Carré, G. A. et al. Loss of p300 and CBP disrupts histone acetylation at the mouse Sry promoter and causes XY gonadal sex reversal. Hum. Mol. Genet. 27, 190-198 (2018).

25. Hecker, M. et al. Terminology of gonadal anomalies in fish and amphibians resulting from chemical exposures. Rev. Environ. Contam. Toxicol. 187, 103-131 (2006).

26. Cheng, H., Guo, Y., Yu, Q. \& Zhou, R. The rice field eel as a model system for vertebrate sexual development. Cytogenet. Genome Res. 101, 274-277 (2003).

27. Szczerbal, l. et al. Disorders of sex development are an emerging problem in french bulldogs: a description of six new cases and a review of the literature. Sex. Dev. 13, 205-211 (2019).

28. De Lorenzi, L. et al. Testicular XX (SRY-Negative) disorder of sex development in cat. Sex. Dev. 11, 210-216 (2017)

29. Major, A. T. \& Smith, C. A. Sex reversal in birds. Sex. Dev. 10, 288-300 (2016).

30. Bullough, W. Hermaphroditism in the lower vertebrates. Nature 160, 9 (1947).

31. Liu, C. K. Rudimentary hermaphroditism in the Symbranchoid eel. Monopterus Javanesis. Sinensia. 15, 1-8 (1944).

32. Stuart, T. \& Satija, R. Integrative single-cell analysis. Nat. Rev. Genet. 20, 257-272 (2019).

33. Wang, S. et al. Single-cell transcriptomic atlas of primate ovarian. Aging Cell. 180, 585-600 (2020).

34. Han, $X$. et al. Construction of a human cell landscape at single-cell level. Nature 581, 303-309 (2020).

35. Becht, E. et al. Dimensionality reduction for visualizing single-cell data using UMAP. Nat. Biotechnol. 37, 38-44 (2019).

36. Miao, L. et al. Translation repression by maternal RNA binding protein Zar1 is essential for early oogenesis in zebrafish. Development 144, 128-138 (2017).

37. Zhu, T., Gui, L., Zhu, Y., Li, Y. \& Li, M. Dnd is required for primordial germ cell specification in Oryzias celebensis. Gene 679, 36-43 (2018).

38. Xiao, Q. et al. Visualizing primordial germ cell migration in embryos of rice field eel (Monopterus albus) using fluorescent protein tagged $3^{\prime}$ untranslated regions of nanos3, dead end and vasa. Comp. Biochem. Physiol. B Biochem. Mol. Biol. 235, 62-69 (2019).

39. Matson, C. K. et al. The mammalian doublesex homolog DMRT1 is a transcriptional gatekeeper that controls the mitosis versus meiosis decision in male germ cells. Dev. Cell. 19, 612-624 (2010).

40. Wu, W. D., Yu, K. W., Zhong, N., Xiao, Y. \& She, Z. Y. Roles and mechanisms of Kinesin-6 KIF20A in spindle organization during cell division. Eur. J. Cell Biol. 98, 74-80 (2019).

41. Kwon, Y. T. \& Ciechanover, A. The Ubiquitin code in the ubiquitin-proteasome system and autophagy. Trends Biochem. Sci. 42, 873-886 (2017).
42. Yasuda, S. et al. Stress- and ubiquitylation-dependent phase separation of the proteasome. Nature 578, 296-300 (2020).

43. de la Cruz, J., Karbstein, K. \& Woolford, J. L. Jr. Functions of ribosomal proteins in assembly of eukaryotic ribosomes in vivo. Annu. Rev. Biochem. 84, 93-129 (2015).

44. Richards, J. S., Ren, Y. A., Candelaria, N., Adams, J. E. \& Rajkovic, A. Ovarian follicular theca cell recruitment, differentiation, and impact on fertility: 2017 update. Endocr. Rev. 39, 1-20 (2018).

45. Aoyama, M. et al. Identification of a new theca/interstitial cell-specific gene and its biological role in growth of mouse ovarian follicles at the gonadotropin-independent stage. Front. Endocrinol. 10, 553 (2019).

46. Raudvere, U. et al. g:Profiler: a web server for functional enrichment analysis and conversions of gene lists (2019 update). Nucleic Acids Res. 47, W191-W8 (2019).

47. Mullen, R. D., Ontiveros, A. E., Moses, M. M. \& Behringer, R. R. AMH and AMHR2 mutations: a spectrum of reproductive phenotypes across vertebrate species. Dev. Biol. 455, 1-9 (2019).

48. Rajakumar, A. \& Senthilkumaran, B. Steroidogenesis and its regulation in teleost-a review. Fish. Physiol. Biochem. 46, 803-818 (2020).

49. Gou, L. T. et al. Ubiquitination-deficient mutations in human Piwi cause male infertility by impairing histone-to-protamine exchange during spermiogenesis. Cell 169, 1090-104.e13 (2017).

50. Wen, L. \& Tang, F. Human germline cell development: from the perspective of single-cell sequencing. Mol. Cell. 76, 320-328 (2019).

51. Zou, K. et al. Production of offspring from a germline stem cell line derived from neonatal ovaries. Nat. Cell Biol. 11, 631-636 (2009).

52. Sohni, A. et al. The neonatal and adult human testis defined at the single-cell level. Cell Rep. 26, 1501-1517. e4 (2019).

53. Cho, C. et al. Haploinsufficiency of protamine-1 or -2 causes infertility in mice Nat. Genet. 28, 82-86 (2001).

54. Sheng, Y. et al. RAB37 interacts directly with ATG5 and promotes autophagosome formation via regulating ATG5-12-16 complex assembly. Cell Death Differ. 25, 918-934 (2017).

55. Dobin, A. et al. STAR: ultrafast universal RNA-seq aligner. Bioinformatics 29, 15-21 (2013).

56. Butler, A., Hoffman, P., Smibert, P., Papalexi, E. \& Satija, R. Integrating single-cell transcriptomic data across different conditions, technologies, and species. Nat. Biotechnol. 36, 411-420 (2018).

57. Qiu, X. et al. Reversed graph embedding resolves complex single-cell trajectories. Nat. Methods 14, 979-982 (2017).

58. Rice, P., Longden, I. \& Bleasby, A. EMBOSS: the European Molecular Biology Open Software Suite. Trends Genet. 16, 276-277 (2000).

59. $\mathrm{Hu}, \mathrm{H}$. et al. AnimalTFDB 3.0: a comprehensive resource for annotation and prediction of animal transcription factors. Nucleic Acids Res. 47, D33-D38 (2019).

60. Buchfink, B., Xie, C. \& Huson, D. H. Fast and sensitive protein alignment using DIAMOND. Nat. Methods 12, 59-60 (2015).

61. Fornes, O. et al. JASPAR 2020: update of the open-access database of transcription factor binding profiles. Nucleic Acids Res. 48, D87-D92 (2020). 